\title{
COUPLED MULTIBODY-MID FIDELITY AERODYNAMIC SOLVER FOR TILTROTOR AEROELASTIC SIMULATION
}

\author{
ALESSANDRO COCCO*, ALBERTO SAVINO*, ALEX ZANOTTI*, \\ ANDREA ZANONI*, PIERANGELO MASARATI* AND VINCENZO \\ MUSCARELLO*
}

*Politecnico di Milano, Milano - Italy

e-mail: alessandro.cocco@polimi.it

Key words: Multiphysics, Coupled Problems, Co-Simulation, Fluid-Structure Interaction, Multibody, Mid-fidelity Aerodynamics

\begin{abstract}
This paper proposes a new aeroelastic solution applicable to fixed and rotarywing aircraft by joining the multibody solver MBDyn and the mid-fidelity aerodynamic tool DUST, through the partitioned multi-physics coupling library preCICE. The coupled MBDyn-DUST simulation environment is intended for the evaluation of performance, loads, and vibratory levels of aircraft of unconventional configuration, such as tiltrotors, during critical transient maneuvers, and to perform aeroelastic stability assessment. The coupling has been tested and validated using simple aeroelastic models available in the literature, and subsequently used to simulate a tiltrotor roll maneuver in airplane mode.
\end{abstract}

\section{INTRODUCTION}

The design of complex flying machines such as tiltrotor aircraft is a challenge for engineers and scientists. For this reason, numerical tools able to simulate the whole machine are needed. Such tools must support the analysis of rather different operating conditions and the simulation of the various parts of the system with the required level of detail. Tiltrotor aircraft must be able to take-off and land as helicopters and, once completed the conversion maneuver, carry on the flight as an airplane. The combination of a multibody solver with a mid-fidelity aerodynamic tool aims at representing an ideal trade-off, to obtain fast and at the same time accurate solutions for the preliminary design of tiltrotor aircraft.

Tiltrotor dynamics are often investigated through a multibody approach, which takes into account the nonlinear dynamics of the interconnected bodies representing the tiltrotor components during the transients $[1,2]$. The multibody approach is also used to investigate aeroelastic phenomena, especially in airplane mode flight where whirl-flutter 
instabilities may occur [3]. In the context of this work, the multibody software selected is MBDyn (http://www.mbdyn.org/, [4]). To simulate transients, an unsteady aerodynamic model is needed that can be coupled to the dynamics of the system. MBDyn supports simple built-in rotorcraft aerodynamics models capable of predicting tiltrotor aeroelastic stability $[5,6]$; however, to simulate tiltrotor maneuvers and estimate aeroelastic loads, aerodynamics based on Blade Element/Momentum Theory (BE/MT) is not sufficiently accurate. No aerodynamic interference between the rotor and the wing is taken into account; this can lead to a significant underestimation of the aerodynamic loads and loss of information related to periodic actions. Coupling with computational fluid dynamics (CFD) solvers was implemented to cope with this limitation $[7,8,9,10,11]$. The downside of this approach relies in the computational cost of such detailed description of the aerodynamics. To overcome this, a good trade-off between efficiency and accuracy consists in coupling with a mid-fidelity aerodynamic solver. With this aim, MBDyn has been combined with the mid-fidelity, fast and reliable aerodynamic solver DUST (https://www.dust-project.org/, [12, 13, 14]). Previous work highlighted the ability of DUST to simulate the complex aerodynamics of an entire tiltrotor vehicle, capturing the interactions between the different parts [15].

The coupling of the two software relies on the partitioned multi-physics coupling library preCICE [16], a very useful and robust togl for managing the communication between different solvers.

This paper describe

MBDyn with the mid

coupled numerical tool is provided by analysint

extensively used as abenchmark for flutter pron

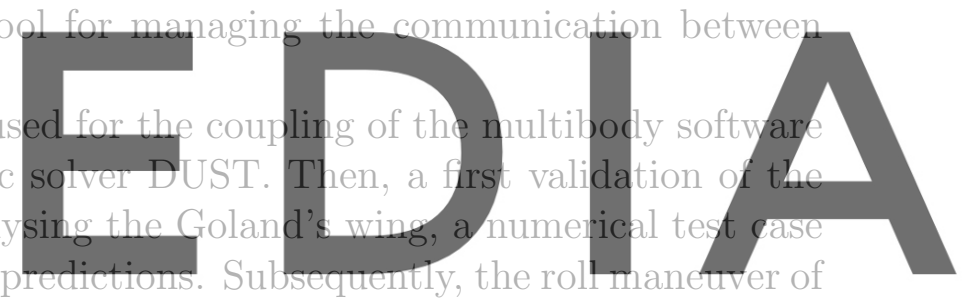

the XV-15 tiltrotor was simulated, showing the effects of the mutual interactions produced

Register for freedatehttps/drwww.scipedia.com to download the version without the watermark

\section{SOFTWARE DESCRIPTION}

\section{$2.1 \quad$ MBDyn}

In the last 20+ years Politecnico di Milano developed a free general purpose multibody software called MBDyn, with the aim of gaining autonomous modeling capabilities of generic problems related to the dynamics of complex aeroelastic systems, specifically rotorcraft and tiltrotor systems. The formulation consists in writing Newton-Euler equations of motion of independent rigid bodies in first order differential form. Thus, the resulting system of Differential-Algebraic Equations (DAEs) is in the form

$$
\begin{aligned}
\mathbf{M}(\mathbf{x}, \mathbf{t}) \dot{\mathbf{x}} & =\mathbf{p} \\
\dot{\mathbf{p}} & =\phi_{/ \mathbf{x}}^{T} \boldsymbol{\lambda}+\mathbf{f}_{i}(\dot{\mathbf{x}}, \mathbf{x}, t)+\mathbf{f}_{e}(\dot{\mathbf{x}}, \mathbf{x}, t) \\
\phi(\mathbf{x}) & =\mathbf{0}
\end{aligned}
$$


where $\mathbf{x}$ are the kinematic unknowns, $\mathbf{p}$ the momentum unknowns, $\boldsymbol{\lambda}$ the algebraic Lagrangian multipliers, $\mathbf{M}$ is a configuration and time dependent inertia matrix, $\mathbf{f}_{i}, \mathbf{f}_{e}$ are arbitrary internal and external forces, $\boldsymbol{\phi}(\mathbf{x})$ are the nonlinear algebraic constraint equations (honolomic constraints) and $\boldsymbol{\phi}_{/ \mathbf{x}}^{T}$ is the Jacobian matrix of the holonomic constraints with respect to the kinematic unknowns. Each node instantiates the writing of balance equations (1b), while only nodes to which inertia properties are associated instantiate the writing of momenta definitions (1a). Additional states, associated with scalar fields (namely, hydraulic pressure, temperature, electric current) and thus the associated differential balance equations, can be taken into account through a specialized set of nodes.

The bodies can be connected either by elastic and viscoelastic internal forces, or by kinematic constraints, which are explicitly added as algebraic equations to form a system of Differential-Algebraic Equations (DAE). Deformable components can be modeled by means of a broad set of elements ranging from lumped (rods, linear and angular springs) to nonlinear Finite Element (FE) beams and shells and Component Mode Synthesis (CMS) elements [17], all allowing a variety of viscoelastic constitutive laws through a generic, extensible interface. Simple aerodynamics can be modeled by internal elements that exploit the Blade Element model by look-up tables of the aerodynamic coefficients.

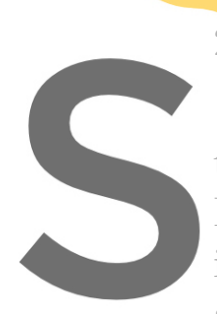

2.2 DUST
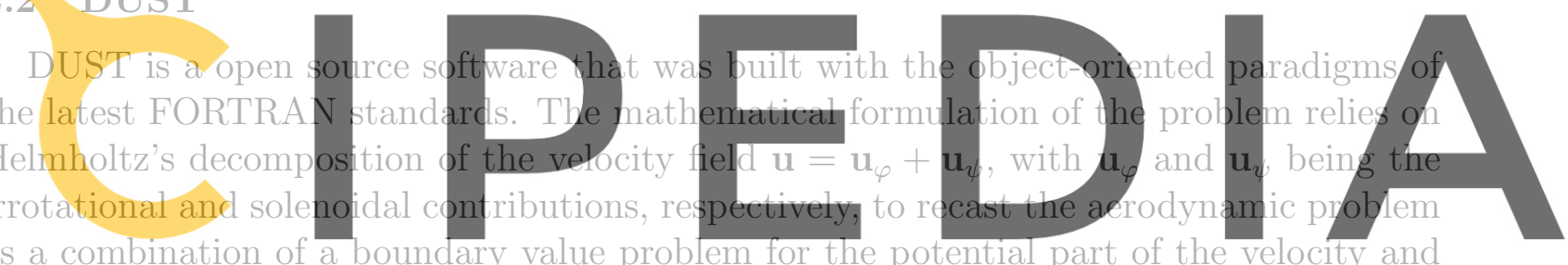

as a combination of a boundary value problem for the potential part of the velocity and

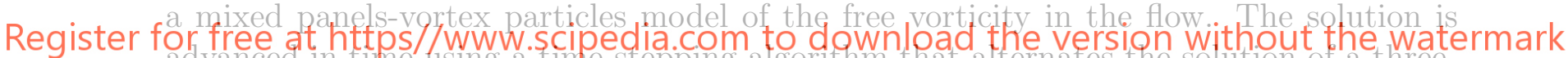
advanced in tine usme a time-stepping algorthm that alternates the solution of a three-

dimensional boundary element method for $\mathbf{u}_{\varphi}$ and the Lagrangian evolution in time of the rotational part of the velocity $\mathbf{u}_{\psi}$. Only the surface mesh of the model is required and different aerodynamic elements allow for different levels of fidelity in the model, ranging from lifting line elements to zero-thickness lifting surfaces and surface panels. A piecewiseuniform distribution of doublets and sources is associated with surface panels, according to a Morino-like formulation for the velocity potential [18]. Thin lifting bodies can also be modelled as zero-thickness surfaces of vortex lattice elements, for which a velocity-based non-penetration condition is assigned.

The wake shed from the trailing edges of lifting bodies is modeled as a panel wake, which shares the same spatial discretization that is used to model the lifting bodies and the same formulation as vortex lattice elements in terms of geometry and singularity distribution. When advected downstream, the panel wake is converted into vortex particles in order to obtain a more robust wake formulation that is suitable for the representation of the interactional aerodynamics of both rotorcraft and complex aircraft configurations. The vortex particles method (VPM) [19, 20] is a Lagrangian grid-free method describing the 
wake evolution through the rotational component of the velocity field $\mathbf{u}_{\psi}$ by means of the material vortex particles used to obtain the approximated vorticity field, namely:

$$
\boldsymbol{\omega}^{h}(\mathbf{r}, t)=\sum_{p=1}^{N_{p}} \boldsymbol{\alpha}_{p}(t) \zeta\left(\mathbf{r}-\mathbf{r}_{p}(t) ; R_{p}\right)
$$

where $\mathbf{r}_{p}(t)$ is the position, $\boldsymbol{\alpha}_{p}(t)$ is the intensity, and $R_{p}$ is the radius of the $p$-th vortex particle, while $\zeta(r)$ is the cut-off function while considering the vorticity distribution that is induced by each particle. By substituting (2) in the equation of the dynamics of vorticity,

$$
\frac{D \boldsymbol{\omega}}{D t}=\boldsymbol{\omega} \cdot \nabla \mathbf{u}+\nu \nabla^{2} \boldsymbol{\omega},
$$

the dynamieal equations for the intensity $\alpha_{p}(t)$ and position $\mathbf{r}_{p}(t)$ of all the material vortex particles to be integrated in time can be obtained, as follows:
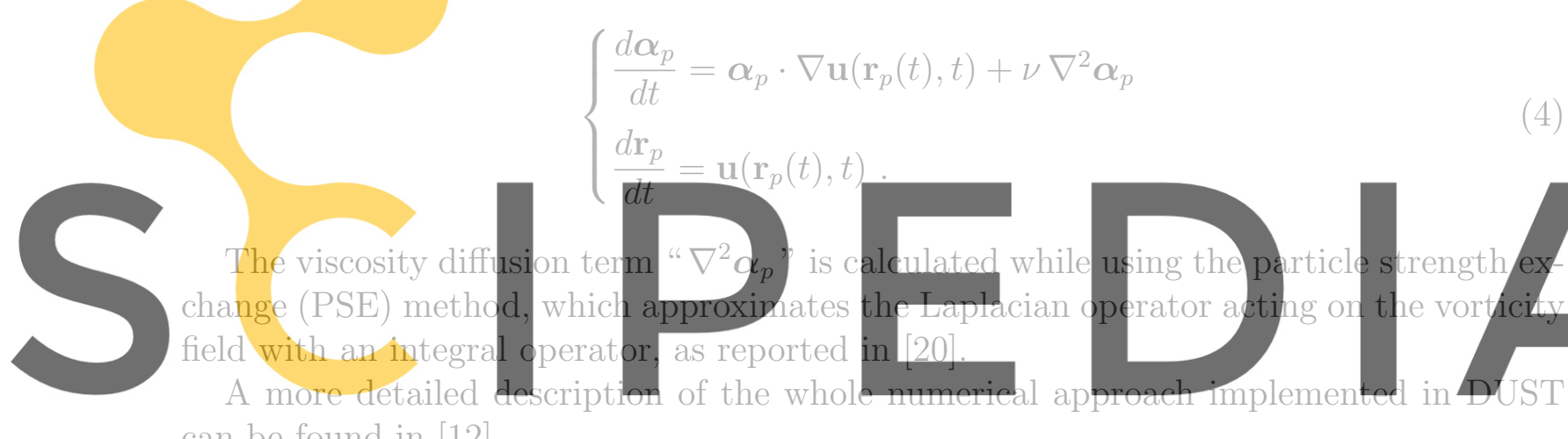

can be found in [12].

Register for free at https//www.scipedia.com to download the version without the watermark 3 COUPLING DESCRIPTION

The communication between DUST and MBDyn is managed by preCICE(Precise Code Interaction Coupling Environment), a coupling library for partitioned multi-physics simulations, originally developed for fluid-structure interaction and conjugate heat transfer simulations. preCICE offers methods for transient equation coupling, communication means, and data mapping schemes. It is written in $\mathrm{C}++$ and offers additional bindings for C, Fortran, Matlab, and Python. preCICE is an open-source software under the LGPL3 license and available on GitHub (https://github.com/precice/). While MBDyn uses its own Application Program Interface (API) for communications with external software without any further modification to the source $\mathrm{C}++$ code, no API was already available in DUST. Few modifications to the source code were required. New Fortran modules collecting all the classes, subroutines and functions required by the adapter for preCICE library were implemented. The optional coupling with external codes is managed through preprocessor directives. Thus, a new adapter has been implemented for allowing the communication of all the kinematic variables (position, orientation, velocity 
and angular velocity) and actions (forces and moments) acting on the nodes of a MBDyn model exposed through an external structural force. In Figure 1 it is shown the communication and information exchange, managed through the two solvers adapters.

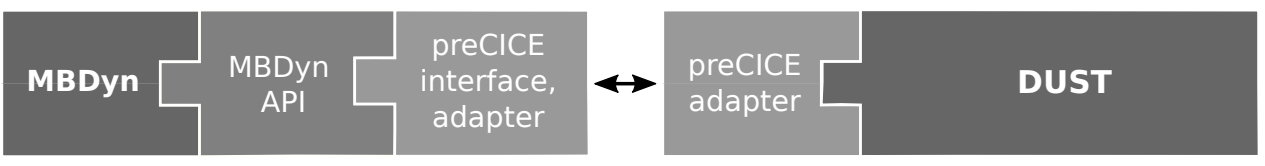

Figure 1: Communication managed through the two solvers adapters.

The interface between the structural and the aerodynamic grids is obtained as a weighted average of the distance between the nodes of the two grids, and used for motion interpolation and the consistent force and moment reduction. Figure 2 shows the nodes of the structural grid $Q$ with their relative reference systems, the centers and the vertices of each aerodynamic mesh elements respectively $P_{e}$ and $P_{p}$, where $q, e$ and $p$ are the corresponding indices.
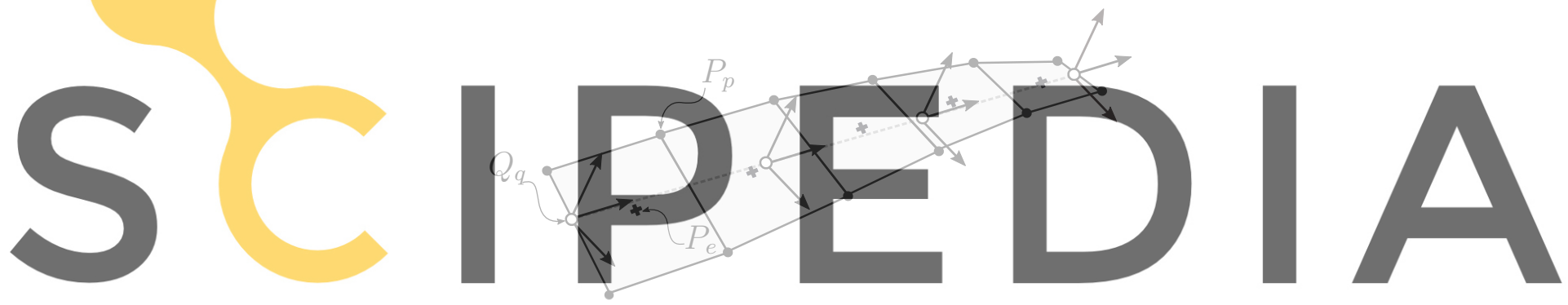

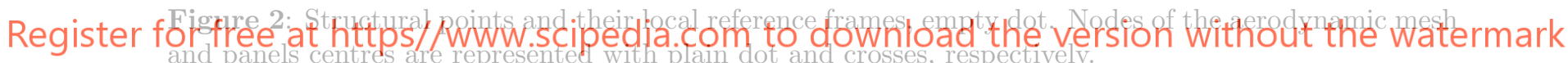

The kinematic variables, $\phi_{p}$, of a point $p$ of the aerodynamic surface of a DUST component is evaluated as the weighted-average,

$$
\phi_{p}=\sum_{q} w_{p q} \phi_{q}
$$

where $\phi_{q}$ is the same kinematic variable associated with the $q$ th structural node of the MBDyn model. Weights $w_{p q}$ could be any set of non-negative real numbers, satisfying the normalization conditions

$$
\sum_{q} w_{p q}=1 \quad, \quad \forall p
$$

since they define the weighted average of the variables associated to the structural nodes $q$ on the aerodynamic nodes $p$. These coefficients could be proportional to some negative power, defined as a user input, of a norm of the vectors $\left(P_{p}-Q_{q}\right)$. As an example, using 
the local coordinates in the reference configuration $\boldsymbol{r}_{p q}$, the norm of these vectors can be defined as,

$$
\left\|\left(P_{p}-Q_{q}\right)\right\|^{2}:=\boldsymbol{r}_{p q}^{T} \mathbf{W} \boldsymbol{r}_{p q},
$$

where $\mathbf{W}$ is a positive (semi-)definite matrix, providing an "anisotropy" degree of freedom to the user in defining the (semi-)norm. Threshold values and maximum number of influencing weights are two criteria, defined as user inputs as well, in order to restrict the average only to the "significant" structural nodes for each aerodynamic point.

\section{Kinematic variables}

The position of a point $P$ in the global reference frame $g$ of the aerodynamic surface is evaluated as,

$$
\left(P_{p}-O\right)^{g}=\sum_{Q \in I_{P}} w_{p q}\left\{\left(Q_{q}-O\right)^{g}+\mathbb{R}_{Q}^{r \rightarrow g}\left(P_{p}-Q_{q}\right)\right\}
$$

where $Q \in I_{p}$ indicates the subset of structural points $Q_{q}$ that belongs to the $I_{p}$ aerodynamic component points, $\left(Q_{q}-O\right)^{g}$ is the distance from the origin of the $Q_{q}$ structural point, and $\mathbb{R}_{Q}^{r \rightarrow g}\left(P_{p}-Q_{q}\right)$ rotates in the global coordinates the distance between the aerodynamic point and the structural one.
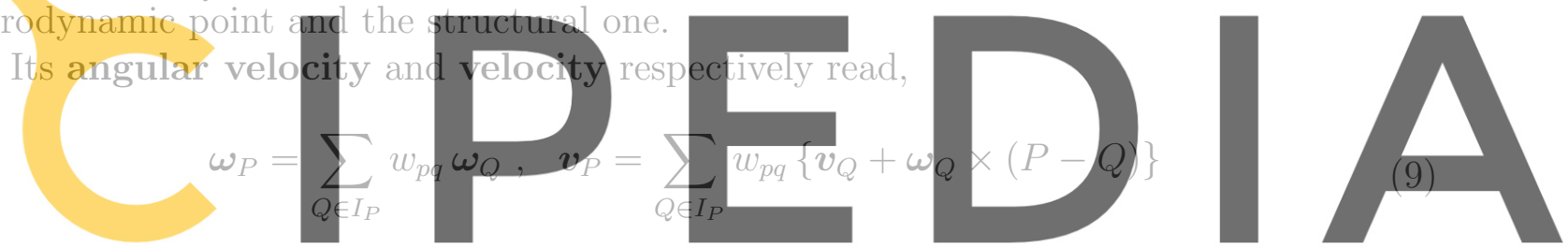

Forces and moments

Register for free at https/fwww.scipedia.com to download the version without the watermark cated at the center of each panels and then transferred to the structural nodes using the summation of forces and transport of moments as follows:

$$
\boldsymbol{f}_{Q}=\sum_{e \in J_{Q}} w_{e q} \boldsymbol{f}_{e} \quad \boldsymbol{m}_{Q}=\sum_{e \in J_{Q}} w_{e q}\left\{\boldsymbol{m}_{e}+\left(P_{e}-Q_{q}\right) \times \boldsymbol{f}_{e}\right\}
$$

where $e \in J_{Q}$ indicated the subset of evaluation points that belongs to each sub-component $J_{Q}$, and the weights $w_{e q}$ are calculated using eqns. 7 and 8 , by taking the distance between each structural node and evaluation points.

\section{Implementation}

Figure 3 shows the flow of information during coupled simulation between the two solvers for an implicit serial scheme. First, the object precice of class t_precice is declared for handling a coupled simulation through preCICE. This object is used both for managing data communication, and for updating coupled components of the aerodynamic 


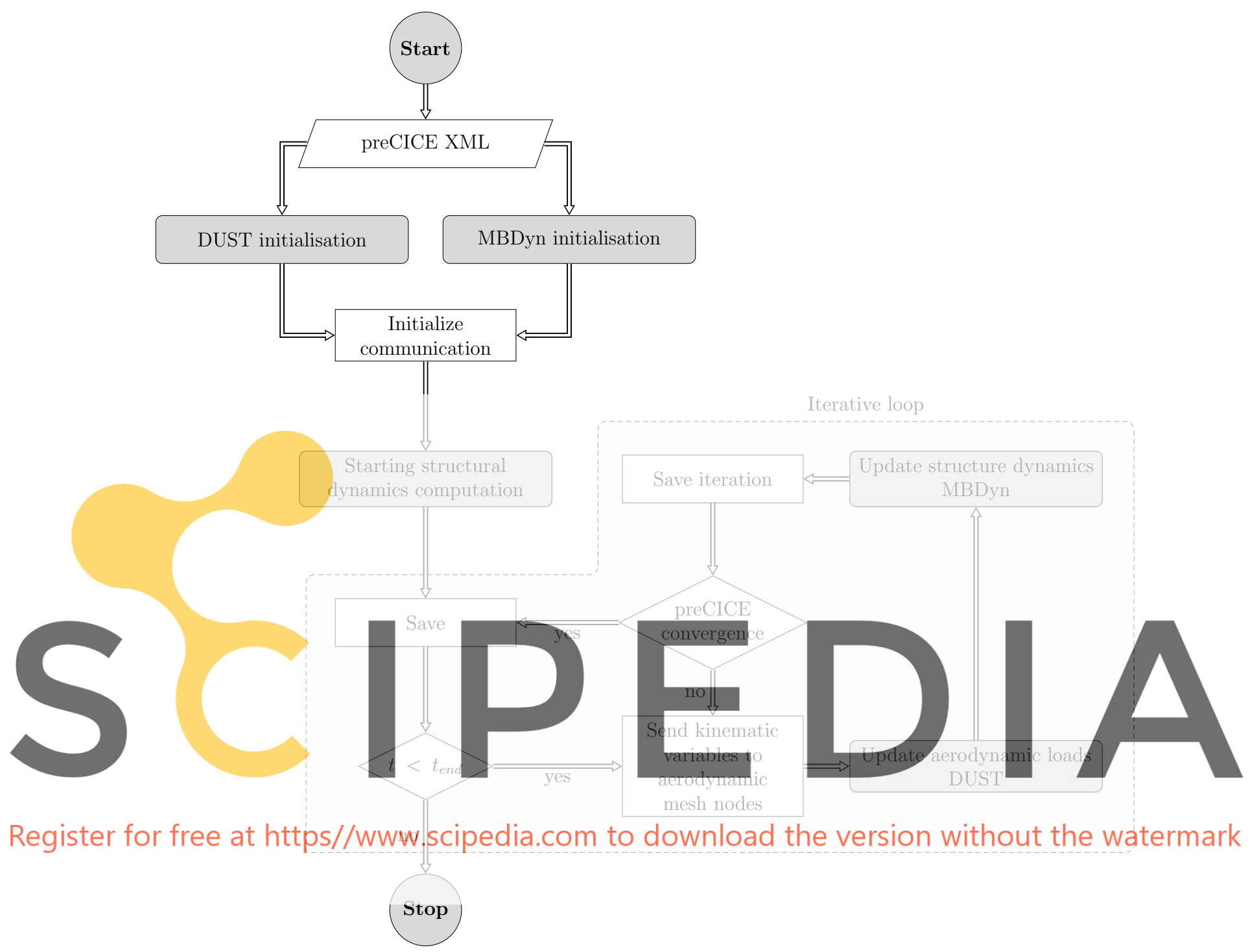

Figure 3: Flowchart of the implicit communication managed by preCICE between DUST and MBDyn

model. Then, DUST participant to the coupled simulation is created, reading XML preCICE configuration file.

After some preliminary operations, the mesh used for coupling the codes is defined and the fields involved in the communication are initialized. Initialization of the Fast Multiple kernels, the wake and the linear system follows.

Before time loop starts, first communication is established between the coupled codes. Time loop starts with the update of DUST explicit aerodynamic elements (lifting lines and actuator disks). A checkpoint of the exchanged fields is stored for being reloaded during sub-iterations of preCICE implicit coupling. Then, DUST receives the kinematic 
variables of the structural nodes from the external software (MBDyn), and updates the surfaces of the coupled components and the near-field wake elements.

Then, the linear system is updated and solved calculating the intensity of the surface panels and vortex lattice elements. The solution of the non-linear lifting line problem follows calculating the circulation $\Gamma$ using tabulated sectional lift and its analytical expression from Kutta-Joukowski theorem. Once the intensity of the surface singularities has been evaluated, surface pressure distribution and elementary forces and moments are retrieved using the Kutta-Joukovsky theorem for the vortex lattice and lifting lines elements, and the unsteady Bernoulli theorem for the 3D-panels.

Aerodynamic forces and moments are reduced to the nodes of the interface between the aerodynamic and structural meshes and sent to MBDyn. Then a convergence check on the kinematics variables follows. If convergence is not reached, the checkpoint fields are reloaded and new sub-iteration begins. If convergence is attained, the time step is finalized saving the status and updating the wake and the geometry of the uncoupled components for the next time step.

\section{VALIDATION AND APPLICATION}

\subsection{Goland's Wing}
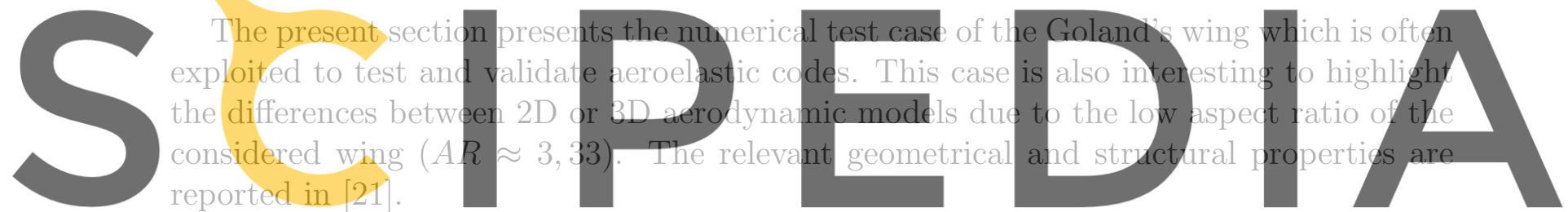

In Figure 4 are reported the results obtained with the coupled codes in terms of fre-

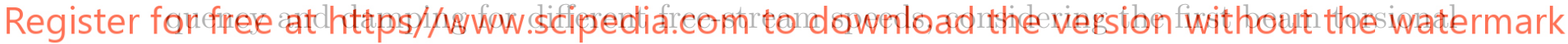
mode. Two different aerodynamic meshes were tested, in one case modeling the wing as a flat plate through vortex lattice (vl) while in the other case modeling it with panels that maintain its shape and thickness. The results obtained in terms of speed and frequency of flutter condition are in line with what is present in the literature with 3D aerodynamic models, as reported in Table 1.

Table 1: Flutter speed of the Goland wing

\begin{tabular}{lccc}
\hline \hline Author & Model & $V_{f}, \mathrm{~m} / \mathrm{s}$ & $\omega_{f}, \mathrm{rad} / \mathrm{s}$ \\
\hline Goland and Luke [22] & Analytical & 137.2 & 70.7 \\
Wang et al. [23] & ZAERO [24] & 174.3 & - \\
Wang et al. [23] & Intrinsic beam + UVLM & 163.8 & - \\
SHARP et al.[25] & Displacement beam + UVLM & 165 & 69 \\
Present Work & DUST (vl)-MBDyn & 168.2 & 68.11 \\
Present Work & DUST(panel)-MBDyn & 171.5 & 69.49 \\
\hline
\end{tabular}


A. Cocco, A. Savino, A. Zanotti, A. Zanoni, P. Masarati and V. Muscarello
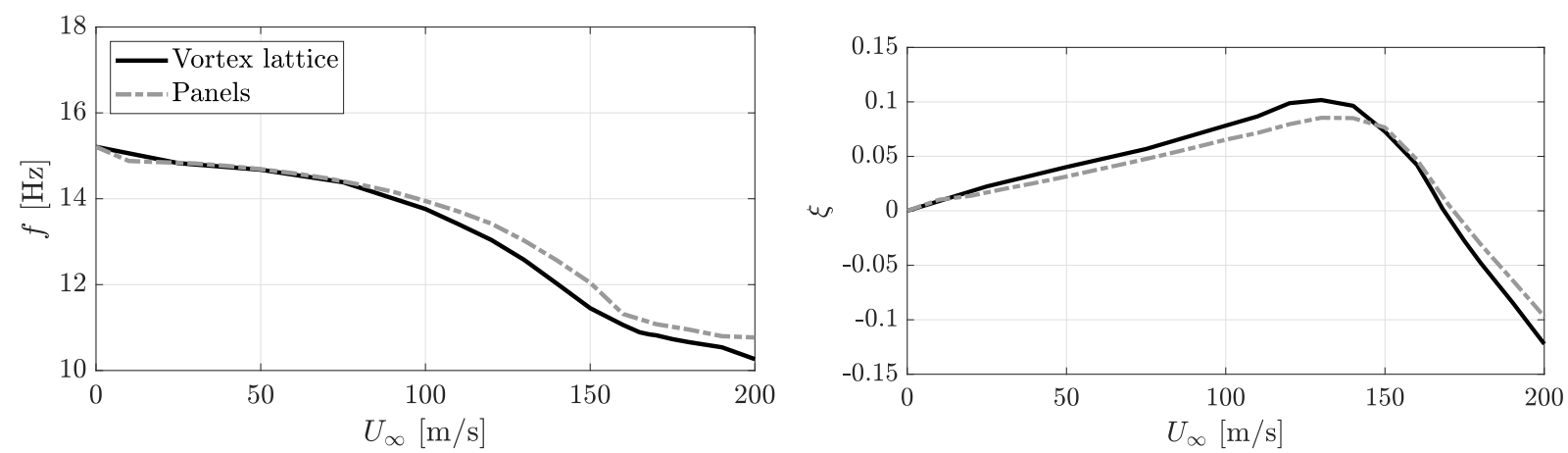

Figure 4: Frequency and damping vs velocity for Goland's wing.

\subsection{Roll Maneuver}

In order to demonstrate the capability of the coupled tool when applied to complex aerodynamics configurations, the complete model of the XV-15 tiltrotor has been tested. The aerodynamic surface mesh is the one investigated in [15] with DUST. For the structural part a MBDyn rigid body model developed with the properties reported by Acree et al. in [26] for the thick wing configuration with the proprotor control chain modeled as

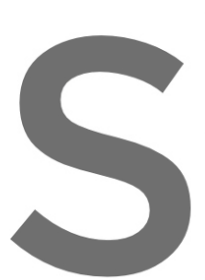
helicopter-like configuration

\section{The simulation was} cruise using a cruise 517 RPM and a collecting
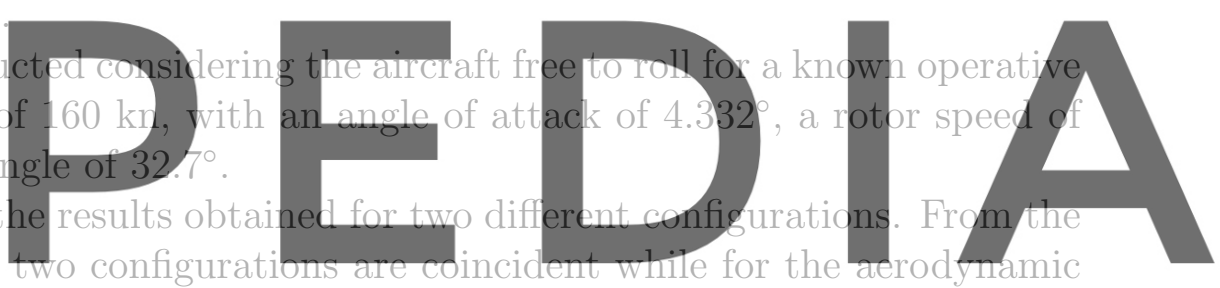

part one solution includes all the surfaces while the other does not consider the aerody-

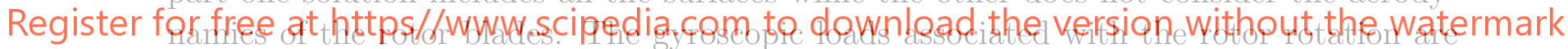
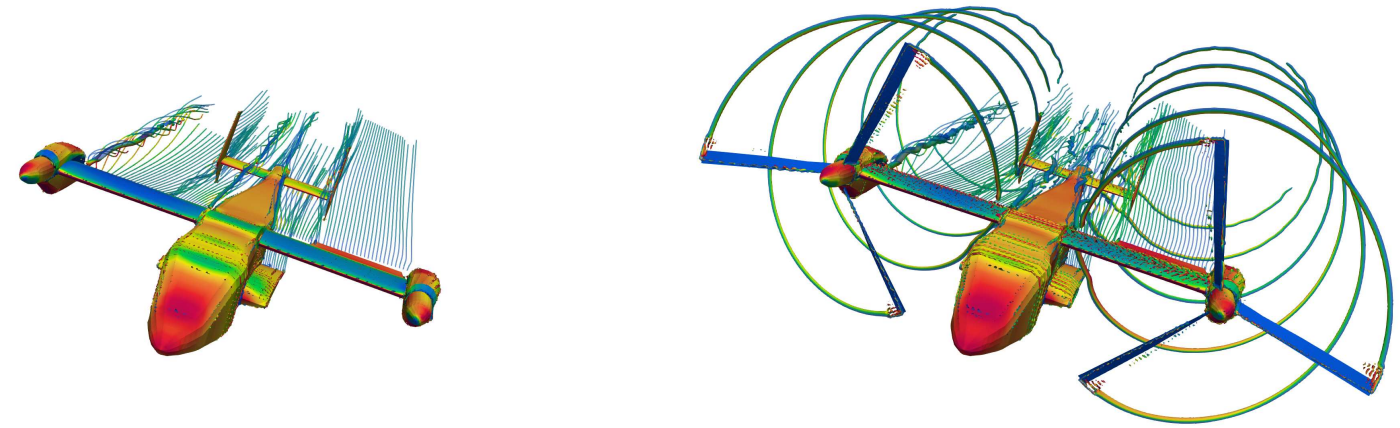

Figure 5: Wake of XV-15 tiltrotor representation for the two simulated configurations.

The difference in the roll maneuver performance due to the aerodynamics of the rotor is not negligible. In particular, the analysis shows how the rotor aerodynamic loads decrease 

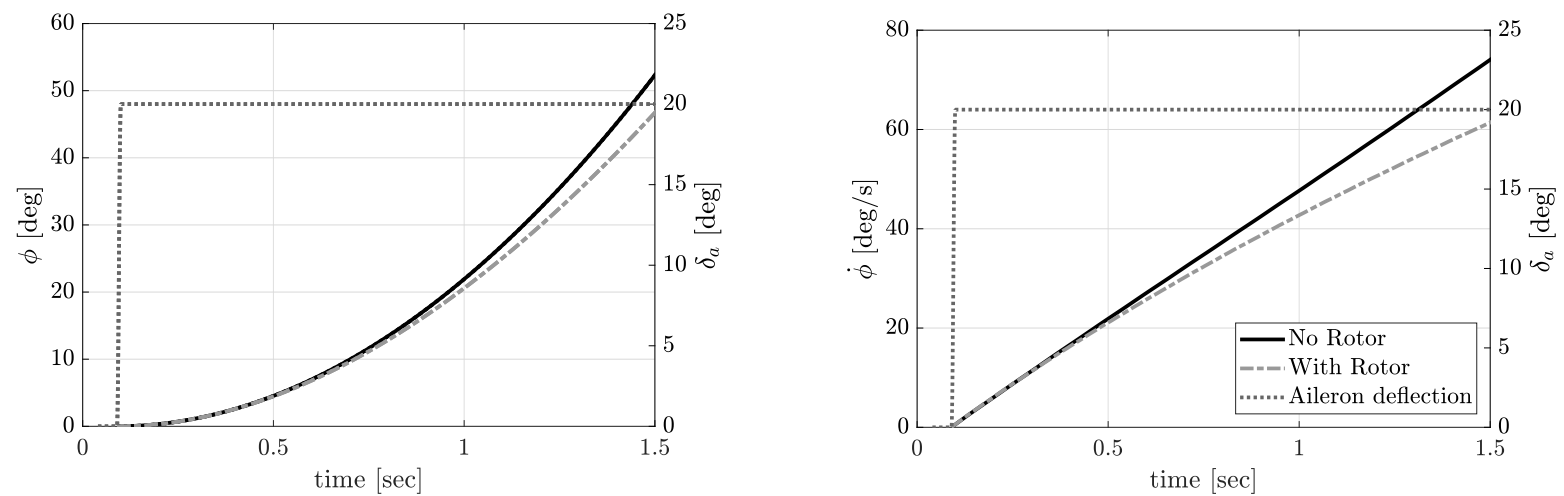

Figure 6: Time history of bank angle $\phi$ and roll rate $\dot{\phi}$ for step ailerons deflection $\delta_{a}$

the roll rate and produces a contrasting effect on the maneuver. This is related to the backward tilting of the rotor induced by the component of reference velocity associated with roll rate in the rotor's plane. Furthermore, the presence of the rotor wake generates a complex flow field, showed in figure 5, that interacts with the tail surfaces.

5 CONCLUSIONS The present work showed possible applications of the developed coupled solver
tween DUST and MBDyn, with the yse of the pre CICE library. The code validation
phase was completed by preliminary application to different types of analysis, including
complex configurations. The maneuvering of a tiltrotor donfiguration has been consid-
ered, as the work is motivated by the need to analyze innovative configurations for the

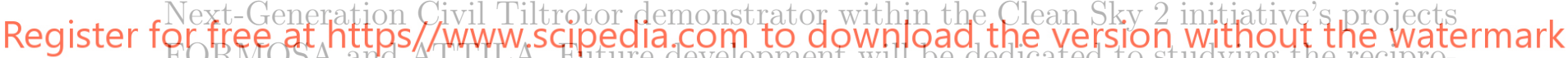
FOR IOSA and AT ITA. Future development will be dedicated to studying the recipro-

cal interactions between the different parts of the aircraft, simulating ever more realistic conditions and by allowing the vehicle to undergo pitch and yaw as well.

\section{ACKNOWLEDGEMENTS}

The research leading to these results has received funding from the Clean Sky 2 - H2020 Framework Programme, under the grant agreement N.885971 (the FORMOSA project), and N.863418 (the ATTILA project).

\section{REFERENCES}

[1] Gian Luca Ghiringhelli, Pierangelo Masarati, Paolo Mantegazza, and Mark W. Nixon. Multi-body analysis of a tiltrotor configuration. Nonlinear Dynamics, 19(4):333-357, August 1999. doi:10.1023/A:1008386219934.

[2] Giuseppe Quaranta, Pierangelo Masarati, Massimiliano Lanz, Gian Luca Ghiringhelli, Paolo Mantegazza, and Mark W. Nixon. Dynamic stability of soft-in-plane tiltrotors by 
parallel multibody analysis. In 26th European Rotorcraft Forum, pages 60.1-9, The Hague, The Netherlands, 26-29 September 2000.

[3] M. Mattaboni, P. Masarati, G. Quaranta, and P. Mantegazza. Multibody simulation of integrated tiltrotor flight mechanics, aeroelasticity and control. J. of Guidance, Control, and Dynamics, 35(5):1391-1405, September/October 2012. doi:10.2514/1.57309.

[4] Pierangelo Masarati, Marco Morandini, and Paolo Mantegazza. An efficient formulation for general-purpose multibody/multiphysics analysis. J. of Computational and Nonlinear Dynamics, 9(4):041001, 2014. doi:10.1115/1.4025628.

[5] J. Shen, P. Masarati, D. J. Piatak, M. W. Nixon, J. D. Singleton, and B. Roget. Modeling a stiff-inplane tiltrotor using two multibody analyses: a validation study. In American Helicopter Society 64th Annual Forum, Montreal, Canada, April 29-May 12008.

[6] Pierangelo Masarati, Vincenzo Muscarello, and Giuseppe Quaranta. Linearized aeroservoelastic analysis of rotary-wing aircraft. In 36th European Rotorcraft Forum, pages 099.1-10, Paris, France, September 7-9 2010.

[7] Giuseppe Quaranta, Giampiero Bindolino, Pierangelo Masarati, and Paolo Mantegazza. Toward a computational framework for rotorcraft multi-physics analysis: Adding computational aerodynamics to multibody rotor models. In 30th European Rotorcraft Forum, pages 18.1-14, Marseille, France, 14-16 September 2004.

[8] Pierangelo Masarati, Giuseppe Quaranta, Massimo Gennaretti, and Jacopo Serafini. Aeroservoelastic analysis of rotorcraft-pilot coupling: a parametric study. In American Helicopter Society 66th Annual Forum, Phoenix, AZ, May 11-13 2010.

[9] L. Cavagna, P. Masarati, and G. Quaranta. Coupled multibody/CFD simulation of maneuvering flexible aircraft. Journal of Aircraft, 48(1):92-106, January-February 2011. doi:10.2514/1.C000253.

[10] P. Masarati and J. Sitaraman. Tightly coupled CFD/multibody analysis of NREL unsteady aerodynamic experiment phase VI rotor. In 49th AIAA Aerospace Sciences Meeting, Orlando, Florida, January 4-7 2011.

[11] Pierangelo Masarati, Giuseppe Quaranta, Massimo Gennaretti, and Jacopo Serafini. An investigation of aeroelastic rotorcraft-pilot interaction. In 37th European Rotorcraft Forum, Gallarate, Italy, September 13-15 2011. Paper no. 112.

[12] Matteo Tugnoli, Davide Montagnani, Monica Syal, Giovanni Droandi, and Alex Zanotti. Mid-fidelity approach to aerodynamic simulations of unconventional vtol aircraft configurations. Aerospace Science and Technology, 115:106804, 2021.

[13] Davide Montagnani, Matteo Tugnoli, Federico Fonte, Alex Zanotti, Giovanni Droandi, and Monica Syal. Mid-fidelity analysis of unsteady interactional aerodynamics of complex vtol configurations. In $45^{\text {th }}$ European Rotorcraft Forum, Warsaw, Poland, September 2019. 
[14] Davide Montagnani, Matteo Tugnoli, Alex Zanotti, Monica Syal, and Giovanni Droandi. Analysis of the interactional aerodynamics of the vahana evtol using a medium fidelity open source tool. In Proceedings of the VFS Aeromechanics for Advanced Vertical Flight Technical Meeting, San Jose, CA, USA, January 21-23 2020. AHS International.

[15] Alex Zanotti, Alberto Savino, Michele Palazzi, Matteo Tugnoli, and Vincenzo Muscarello. Assessment of a mid-fidelity numerical approach for the investigation of tiltrotor aerodynamics. Applied Sciences, 11(8):3385, 2021.

[16] Hans-Joachim Bungartz, Florian Lindner, Bernhard Gatzhammer, Miriam Mehl, Klaudius Scheufele, Alexander Shukaev, and Benjamin Uekermann. preCICE - a fully parallel library for multi-physics surface coupling. Computers and Fluids, 141:250-258, 2016. Advances in Fluid-Structure Interaction.

[17] Roy R. Craig, Jr. and Mervyn C. C. Bampton. Coupling of substructures for dynamic analysis. AIAA Journal, 6(7):1313-1319, July 1968.

[18] Luigi Morino and Ching-Chiang Kuot. Subsonic potential aerodynamics for complex configurations: a general theory. AIAA Journal, 12(2):191-197, 1974.

[19] Georges-Henri Cottet, Petros D Koumoutsakos, D Petros, et al. Vortex methods: theory and practice. Cambridge University Press, 2000.

[20] Gregoire Stephane Winckelmans. Topics in vortex methods for the computation of threeand two-dimensional incompressible unsteady flows. $\mathrm{PhD}$ thesis, California Institute of Technology, 1989.

[21] Mayuresh J Patil, Dewey H Hodges, and Carlos ES Cesnik. Nonlinear aeroelastic analysis of complete aircraft in subsonic flow. Journal of Aircraft, 37(5):753-760, 2000.

[22] Martin Goland. The flutter of a uniform cantilever wing. Journal of Applied MechanicsTransactions of the Asme, 12(4):A197-A208, 1945.

[23] Zhicun Wang, PC Chen, DD Liu, DT Mook, and MJ Patil. Time domain nonlinear aeroelastic analysis for hale wings. In 47 th AIAA/ASME/ASCE/AHS/ASC Structures, Structural Dynamics, and Materials Conference 14th AIAA/ASME/AHS Adaptive Structures Conference 7th, page 1640, 2006.

[24] Zaero user's manual. Technical Report Ver. 8.3, ZONATechnology, Inc., Scottsdale, AZ, 2008.

[25] Joseba Murua, Rafael Palacios, and J Michael R Graham. Assessment of wake-tail interference effects on the dynamics of flexible aircraft. AIAA Journal, 50(7):1575-1585, 2012.

[26] C. W. Acree, R. J. Peyran, and Wayne Johnson. Rotor design options for improving tiltrotor whirl-flutter stability margins. Journal of the American Helicopter Society, 46(2):87-95, April 2001. doi:10.4050/JAHS.56.022004. 